EPJ manuscript No.

(will be inserted by the editor)

\title{
Unconventional Quantum Phases in Lattice Bosonic Mixtures
}

P. Buonsante ${ }^{1}$, S.M. Giampaolo ${ }^{2,3}$, F. Illuminati ${ }^{2,3,4}$, V. Penna ${ }^{1}$, and A. Vezzani ${ }^{5,6}$

${ }^{1}$ C.N.I.S.M. and Dipartimento di Fisica, Politecnico di Torino, C.so Duca degli Abruzzi 24, I-10129 Torino, Italy.

2 Dipartimento di Matematica e Informatica, Università degli Studi di Salerno, Via Ponte don Melillo, I-84084 Fisciano (SA), Italy.

3 CNR-INFM Coherentia, Napoli, Italy, CNISM Unità di Salerno, and INFN Sezione di Napoli, Gruppo collegato di Salerno, Baronissi (SA), Italy.

${ }^{4}$ Institute for Scientific Interchange, Viale Settimio Severo 65, I-10133 Torino, Italy.

${ }^{5}$ CNR-INFM, S3, National Research Center, via Campi 213/a, I-41100 Modena.

${ }^{6}$ Dipartimento di Fisica, Università degli Studi di Parma, V.le G.P. Usberti n.7/A, I-43100 Parma, Italy

\begin{abstract}
We discuss strongly interacting one-dimensional boson-boson mixtures and study their quantum phases as the interspecies repulsion is increased. In particular, we analyze the low-energy quantum emulsion metastable states occurring at large values of the interspecies interaction, which are expected to prevent the system from reaching its true ground state. A significant decrease in the visibility of the atomic clouds is predicted as well, and related to the trapping of the system in the spontaneously disordered quantum emulsion states.
\end{abstract}

\section{Introduction}

In recent years the study of systems of ultracold atoms loaded into optical lattices has drawn much attention because of their value as experimental probes for the investigation of models of condensed matter physics exhibiting key properties of complex many-body systems. One of the most relevant examples in this sense is certainly the Bose-Hubbard model, originally introduced by Haldane 1 as a simple yet interesting variant of the better known fermionic Hubbard model, and later investigated at length and proposed for the description of superfluid ${ }^{4} \mathrm{He}$ trapped in porous media in a seminal paper by M. P. A. Fisher et al. 2]. The observation of the distinctive superfluid-Mott insulator quantum phase transition in a system of ultracold rubidium atoms [3] brilliantly demonstrated the prediction that, under suitable conditions, ultracold bosonic atoms trapped in optical lattices could provide a physical realization of the Bose-Hubbard model 4. The impressive control in atom cooling and trapping soon allowed the realization of more general Bose-Hubbard Hamiltonians, including, e.g., disordered local potentials 566.

The quest for novel and unconventional quantum phases has recently pushed the interest towards even more general variants of the Hubbard model, involving mixtures of particles obeying either the same or different statistics. Beyond their theoretical appeal, these systems are relevant to interesting applications such as implementation of disordered systems [7/8, association of dipolar molecules [9, schemes for quantum computation [10] and realization of quantum spin chains and arrays [11/12.

So far, most of the experiments on lattice-atom mixtures have involved atoms obeying different statistics. Several experimental realizations of lattice Bose-Fermi mixtures have been engineered 8 813]14, whereas, to the best of our knowledge, the first experiment on mixtures 
involving two different bosonic species has been carried out very recently at LENS, in Florence [15.

At the theoretical level, various investigations have been carried out on lattice Bose-Fermi 16 17 18 19 2021] as well as Bose-Bose mixtures [22 23|24|25 26 27]. This wealth of work demonstrates that a comprehensive and systematic study of the phases of atomic lattice mixtures requires a significant analytic and synthetic effort. Indeed, one should be aware that even in the ideal case of a homogeneous lattice, the Hamiltonian describing a bosonic mixture contains five parameters, namely the two intra-species interaction strengths, the inter-species interaction strength and one hopping amplitude for each species. Moreover, the possible phases depend on many physical parameters such as, e.g., the populations of the two atomic species and their commensurability with the lattice size.

For the above reasons, we confine the present analysis to a rather limited region of the phase diagram of a boson-boson mixture, which however encompasses a fair wealth of quantum phases effects. Specifically, we consider two different bosonic species loaded in a 1-D homogeneous lattice, and vary only the interaction among them.

We assume that one of the species is soft-core and at unitary filling, whereas the other is hard-core and at filling 2/5. Our choice is similar to that considered in Refs. [28 29], where the ground state of a Bose-Fermi mixture on a 1-D lattice is analyzed by means of quantum Monte Carlo simulations. Recall indeed that on 1D lattices hard-core bosons and spinless fermions share many features. While we find a qualitatively similar phase diagram, our analysis differs from that in Ref. 29] at least in two respects. Firstly, we adopt a less quantive yet more computationally affordable approach.

More interestingly, we focus on the configurations that emerge at strong values of interspecies interactions. We find that for sufficiently large interspecies interactions the system supports a large number of low energy metastable quantum emulsion states exhibiting glassy features despite the complete absence of any source of disorder in the Hamiltonian parameters. Such a complex energy landscape for lattice bosonic mixtures was pointed out in Ref. [26] and further discussed in Ref. [27] in the presence of the inhomogeneous local potential typical of realistic setups. Interesting related results are presented in Ref. [24] based on a multi-orbital Gross-Pitaevskii approach, while a phase diagram for metastable states is derived in Ref. [30] for dipolar bosons. We carry the analysis of quantum emulsions further on, discussing the arrangement and features of the droplets, as well as the interference pattern obtained after free-expansion of the atomic cloud.

The plan of the paper is as follows: In Sec. 2 we recall the Hamiltonian of the system as well as the mean-field approach we adopt. After the introduction of the quantities characterizing the quantum phases occurring in the system, our results are presented in Sec. 3. In particular, we focus on the behavior of all the aforementioned quantities as functions of the interspecies interaction. As we mention, particular attention is devoted to the quantum emulsion states characterizing the phase-separated regimes. Interestingly we evidence a phase where the softcore bosons behave as a disordered superfluid featuring a global phase coherence but a spatially inhomogeneous density. We conclude with a brief summary of our results.

\section{The System}

The arguments of Ref. [4] can be generalized to a lattice loaded with atoms of two different bosonic species. Under suitable conditions, the system is described by the two-flavor BoseHubbard Hamiltonian

$$
H=U_{12} \sum_{i} n_{1, i} n_{2, i}+\sum_{f} \sum_{i}\left[\frac{U_{f}}{2} n_{f, i}\left(n_{f, i}-1\right)-J_{f}\left(a_{f, i}^{\dagger} a_{f, i+1}+\text { h.c. }\right)\right],
$$

where the lattice boson operators $a_{f, i}^{\dagger}, a_{f, i}$, and $n_{f, i}=a_{f, i}^{\dagger} a_{f, i}$, create, destroy and count atoms of the flavor, or species, $f$ at site $i$. The parameters $U_{f}$ and $U_{12}$ quantify the repulsive interaction between atoms of the same or different species, respectively (henceforth intra- and inter- species 
repulsion). The possibly different hopping amplitude of the two species is quantified by the parameters $J_{f}$. Two-species Bose-Hubbard Hamiltonians similar to the one in Eq. (11) have been considered previously in several works, possibly referring to different internal states of the same bosonic species 23 to spin-1 313233 or dipolar bosons 934.

Since we are in a strong interaction regime, we assume that the state of the system $|\Psi\rangle$ has a Gutzwiller factorized form,

$$
|\Psi\rangle=\prod_{i}\left|\psi_{i}\right\rangle, \quad\left|\psi_{i}\right\rangle=\sum_{p_{1}, p_{2}} \frac{c_{p_{1}, p_{2}}^{(i)}}{\sqrt{p_{1} ! p_{2} !}}\left(a_{1, i}^{\dagger}\right)^{p_{1}}\left(a_{2, i}^{\dagger}\right)^{p_{2}}|\emptyset\rangle
$$

where $|\emptyset\rangle$ denotes the vacuum state, $a_{f, i}|\emptyset\rangle=0$ for any $i$. A time-dependent variational procedure [35] shows that the complex coefficients $c_{p_{1}, p_{2}}^{(j)}$ obey a semiclassical dynamics of the form 9]

$$
\begin{aligned}
i \dot{c}_{p_{1}, p_{2}}^{(j)} & =\left[\frac{U_{1}}{2} p_{1}\left(p_{1}-1\right)+\frac{U_{2}}{2} p_{2}\left(p_{2}-1\right)+U_{1,2} p_{1} p_{2}\right] c_{p_{1}, p_{2}}^{(j)}-J_{1}\left[\sqrt{p_{1}+1} c_{p_{1}+1, p_{2}}^{(j)} \bar{\Phi}_{1}^{(j)}\right. \\
& \left.+\sqrt{p_{1}} c_{p_{1}-1, p_{2}}^{(j)} \Phi_{1}^{(j)}\right]-J_{2}\left[\sqrt{p_{2}+1} c_{p_{1}, p_{2}+1}^{(j)} \bar{\Phi}_{2}^{(j)}+\sqrt{p_{2}} c_{p_{1}, p_{2}-1}^{(j)} \Phi_{2}^{(j)}\right] \\
\Phi_{1}^{(j)} & =\sum_{\ell \sim j} \sum_{p_{1}, p_{2}} \sqrt{p_{1}} \bar{c}_{p_{1}, p_{2}}^{(\ell)} c_{p_{1}+1, p_{2}}^{(\ell)} \quad \Phi_{2}^{(j)}=\sum_{\ell \sim j} \sum_{p_{1}, p_{2}} \sqrt{p_{2}} \bar{c}_{p_{1}, p_{2}}^{(\ell)} c_{p_{1}, p_{2}+1}^{(\ell)}
\end{aligned}
$$

where the symbols ${ }^{-}$and $\sim$ denote complex conjugation and nearest-neighborhood, respectively, and the quantities in Eq. (44) are shorthand notations to simplify Eq. (3).

The ground-state of the system is the lowest-energy normal mode of Eqs. (3), which can be found as the ground state of the mean-field Hamiltonian

$$
\begin{aligned}
\mathcal{H} & =\sum_{i, f}\left\{\frac{U_{f}}{2} n_{f, i}\left(n_{f, i}-1-\mu_{f}\right)-J_{f}\left[a_{f, i}^{\dagger}\left(\alpha_{f, i+1}+\alpha_{f, i-1}\right)+a_{f, i}\left(\bar{\alpha}_{f, i+1}+\bar{\alpha}_{f, i-1}\right)\right]\right\} \\
& +U_{12} \sum_{i} n_{1, i} n_{2, i}
\end{aligned}
$$

subject to the self consistency constraint $\alpha_{f, j}=\left\langle\Psi\left|a_{f, j}\right| \Psi\right\rangle=\left\langle\psi_{j}\left|a_{f, j}\right| \psi_{j}\right\rangle$. Note indeed that the eigenstates of Hamiltonian (5) have the form in Eq. (2). A few points are worth observing here. First of all, the explicit presence of the (species-specific) chemical potentials $\mu_{f}$, which act as Lagrange multipliers fixing the total populations $N_{f}$. This is explicitly needed since Hamiltonian (5) does not commute with the total populations $N_{f}=\sum_{j} n_{f, j}$, unlike Hamiltonian (1). Second, the mean-field theory can be equivalently obtained from Eq. (1) by means of the so-called decoupling approximation, consisting in the substitution $a_{f, i}^{\dagger} a_{f, j} \longrightarrow a_{f, i}^{\dagger} \alpha_{f, j}+\bar{\alpha}_{f, i} a_{f, j}-\bar{\alpha}_{f, i} \alpha_{f, j}$. More interestingly, we note that the above approach treats the on-site terms of Hamiltonian (1) exactly. This in particular means that the cross-correlation function $C_{1,2}$ introduced in Eq. (77) below makes sense also for our decoupled trial state (2). We emphasize the explicit site dependence of our method. That is, unlike earlier works adopting the Gutzwiller approximation 2223136 , we do not force translational invariance on Eq. (2), and hence do not end up with an effective single site theory, which would be unable to describe the structure of phase-separated or generically inhomogeneous configurations. Since we are interested in the effects of an increasing $U_{1,2}$ on a given system, we do not look for the ground state of Hamiltonian (5) at fixed values of the chemical potentials, as it is usually done. Rather, we adjust the $\mu_{1}$ and $\mu_{2}$ so that we obtain the desired fillings. This procedure, by the way, avoids the so-called species depletion problem affecting single-site theories [23/36].

The quantities $\alpha_{j, f}$, referred to as local order parameters, are directly related to several interesting physical quantities, such as the one-body density matrix - related in turn to the condensate fraction of the system and to the interference pattern observed in experiments and the superfluid fraction. 
In the simple case of a single-species homogeneous lattice one recognizes two phases, corresponding to the local order parameters being all zero or all finite. These situations are readily identified with the insulating and superfluid phases, respectively. The resulting phase diagram turns out to be qualitatively correct, and its quantitative agreement with the exact result improves with increasing dimensionality of the lattice.

Being an approximation, the mean-field approach suffers from some limitations. For instance, site decoupling makes the correlation between two sites of a homogeneous lattice independent of their distance, which results in the superfluid phase being always condensate. This clearly does not apply to 1D lattices, where the power law decay of the two-site correlations prevents long range order in the thermodynamic limit. Despite this artifact, the mean-field approach proves useful also on one dimensional systems. Indeed, at the finite - however large - experimentally relevant sizes, the mean-field prediction provides an acceptable approximation to the slow decay of the exact correlations. This for instance results in qualitatively correct interference patterns, characterized by a sharp peak at zero momentum 37. Such a peak is completely washed out in the Mott-insulating regime.

Furthermore, the local nature of the mean-field order parameters allows for configurations where different portions of the system exhibit different phases, thus proving effective in capturing the geometric fluctuations that add to the quantum ones in the presence of inhomogeneity. Different phase domains typically occur in the presence of site-dependent (possibly random) local potentials, but are possible also on homogeneous lattices when the translation invariance is spontaneously broken.

The comparison of the results presented here and in Ref. [27] with those in Refs. [26,29] is a clear evidence of the validity of the qualitative picture provided by the mean-field approach.

For the sake of completeness it should be mentioned that the description of a boson-boson mixture such that both of the atomic species are in a Mott state reveals a further limitation of the mean-field approach. In this case an unrealistic degeneracy for the ground state of the system is predicted, which can be resolved only taking into account second-order quantum fluctuations $[22,36]$. However, the conditions for this artifact to occur are rather specific (the total boson population has to be commensurate with the lattice size) and are never considered in the following.

\section{Results}

The zero-temperature phases of the system are determined by the properties of the ground state of Hamiltonian Eq. (11). We characterize such properties making use of several quantities. The superfluidity of each species can be estimated as the stiffness under phase variations [20138139]

$$
F_{\mathrm{s}}^{(f)}=\lim _{\theta \rightarrow 0} \frac{1}{t} \frac{E(\theta)-E(0)}{N_{f} \theta^{2}}
$$

where $E(\theta)$ is the ground state energy of the Hamiltonian obtained from Eq. (11) by the substi-

tution $a_{f, j}^{\dagger} a_{f, j+1}+a_{f, j} a_{f, j+1}^{\dagger} \longrightarrow e^{i \theta} a_{f, j}^{\dagger} a_{f, j+1}+e^{-i \theta} a_{f, j} a_{f, j+1}^{\dagger}$ in the hopping term of species $f$, while $N_{f}=\sum_{j}\left\langle n_{f, j}\right\rangle$ is the population of the same species. The introduction of the so-called Peierls phases [40] is equivalent to the imposition of twisted boundary conditions.

Two further quantities will be useful in the characterization of the phases we are going to encounter, namely the creation-annihilation cross correlation functions and the local density fluctuation

$$
C_{1,2}=\frac{1}{M} \sum_{i, f}\left(\left\langle a_{f, i}^{\dagger} a_{\bar{f}, i}\right\rangle-\left\langle a_{f, i}^{\dagger}\right\rangle\left\langle a_{\bar{f}, i}\right\rangle\right), \quad \Delta n_{f}=\frac{1}{M} \sum_{i}\left(\left\langle n_{f, i}\right\rangle\right)^{2}-\left(\frac{1}{M} \sum_{i}\left\langle n_{f, i}\right\rangle\right)^{2} .
$$

where the operation ${ }^{-}$exchange the two species, i.e. $\bar{f}=1,2$ if $f=2,1$ respectively. The first quantity is specific to mixtures, accounting for nontrivial local quantum correlations between particles of different kind and, as we observed in the previous section, is well posed for the 

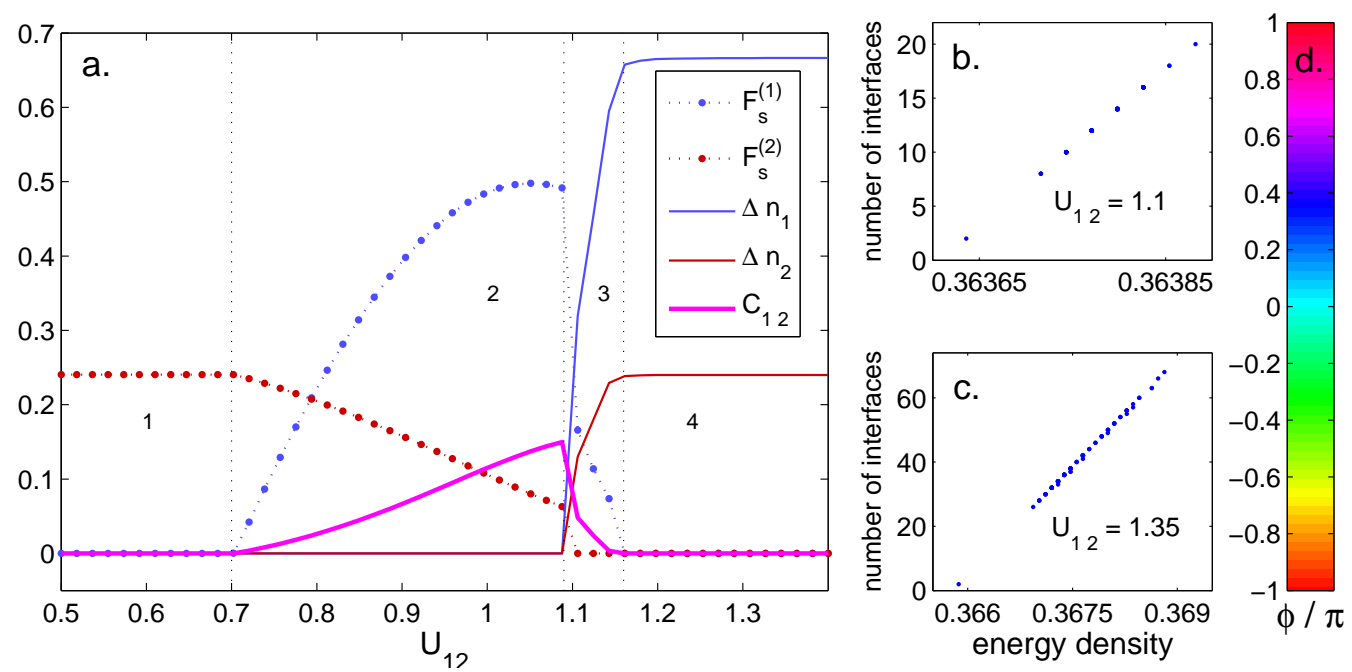

Fig. 1. a. Phase diagram of the system as obtained for a lattice with $M=2000$. The plotted quantities, discussed in Sec. 3. allow us to identify four different quantum phases; Panels b. and c. refer to regions 3 and 4 of the phase diagram, respectively, and demonstrate the linear relation between the system energy and the number of phase interfaces in the quantum emulsion states the system gets trapped into. The panels show 100 data obtained for a lattice with 600 sites. Panel d. shows the color code for the phase of the local order parameter characterizing the emulsion droplets in Fig. 2 .

Gutzwiller trial state in Eq. (21). The second quantity is sensitive to spatial inhomogeneity, and hence it is useful in the characterization of the quantum emulsion states appearing at large interspecies interactions. Also, it conveniently signals phase separation which, in the meanfield approximation, occurs through the emergence of ground states breaking the translational symmetry of the Hamiltonian due to nonlinear effects. The expected homogeneous ground state is recovered as a symmetric superposition of the set of degenerate symmetry-breaking states obtained from each other by a lattice translation.

Finally, the interference pattern in the experimental absorption images of each species is basically determined by the Fourier transform of the relevant one-body density matrix summarizing the first-order correlations [41,

$$
S_{f}(\mathbf{k})=\frac{1}{M} \sum_{j \ell} e^{i \mathbf{k} \cdot\left(\mathbf{r}_{j}-\mathbf{r}_{\ell}\right)} \rho_{j \ell}^{(f)}, \quad \rho_{j \ell}^{(f)}=\left\langle a_{f, i}^{\dagger} a_{f, j}\right\rangle
$$

where $\mathbf{r}_{j}$ is the spatial position of the $j$-th optical lattice site, $\mathbf{k}$ is the (momentum) coordinate in the absorption image. As we will illustrate shortly, these results provide a reasonable description of the system, despite the above-mentioned artifact prediction of a finite condensate fraction.

As we mention above, we focus our attention on a combination of Hamiltonian parameters where several different and interesting phases take over as the interspecies repulsion $U_{1,2}$ is increased. As to the remaining parameters, we choose $U_{1}=1$ as our energy scale, $U_{2}=\infty$ (hardcore bosons), $J_{1}=J_{2}=0.05, N_{1} / M=1$ and $N_{2} / M=0.4$. In order to rule out contributions arising from geometric inhomogeneity we choose a homogeneous lattice comprising $M$ sites, with periodic boundary conditions.

The above described quantities allow us to identify 4 different phases for the system, as it is clear from Fig. 1 When $U_{1,2}$ is sufficiently small the situation is similar to the non-interacting limit $U_{1,2}=0$, in which species 2 is superfluid and species 1 , being strongly interacting and at unitary filling, is in a Mott-insulating state. Since the two species are virtually noninteracting, the cross-correlation $C_{1,2}$ vanishes. The different quantities characterizing the system are independent of $U_{1,2}$ and each species behaves as if it was alone in the lattice. In this situation 
there are no density fluctuations, as it is expected for single-species systems on homogeneous lattices.

As soon as the interspecies interaction exceeds a first critical value, $U_{1,2}^{\prime} \approx 0.7$, it becomes sufficiently strong to induce a nonzero cross correlation. The interaction with the hard-core bosons melts the Mott insulator, and the atoms of species 1 enter a superfluid phase. This can be intuitively explained by observing that, owing to the large interspecies interaction, the simultaneous presence of two atoms of different species on the same site is energetically unfavourable. Hence the atoms of species 1 are so-to-say expelled from the sites occupied by hard-core bosons, and give rise to a nonzero superfluid fraction. Since $U_{1}$ is still sufficiently larger than $U_{1,2}$, the bosons of both species remain delocalized and homogeneous, as demonstrated by the vanishing of $\Delta n_{f}$. It is interesting to observe that the increase of $F_{\mathrm{s}}^{(1)}$ in this phase corresponds to a decrease of $F_{\mathrm{s}}^{(2)}$, as if the hard-core bosons felt the presence of soft-core bosons like a viscous medium slowing down their motion through the lattice.

When the interspecies interaction exceeds a second critical value, $U_{1,2}^{\prime \prime} \approx 1.09$, the mean-field ground-state of the system breaks the translational symmetry of Hamiltonian (5), which signals the occurrence of phase-separation. This is recognized by the nonzero value of the average local density fluctuations of both species. As we mention above, the expected homogeneous density is recovered through a symmetrization of the symmetry-breaking state. As long as the interspecies interaction does not exceed the further critical value $U_{1,2}^{\prime \prime \prime} \approx 1.16$, the two atomic species remain correlated, $C_{1,2}>0$, and coherent. However, while the soft-core bosons are superfluid, the hardcore bosons loose their superfluidity, $F_{\mathrm{s}}^{(2)}=0$. For $U_{1,2}>U_{1,2}^{\prime \prime \prime}$ both $F_{\mathrm{s}}^{(1)}$ and $C_{1,2}$ vanish: the system has reached a completely phase-separated state where no atoms of species 1 are found at sites hosting hard-core bosons, and vice-versa.

The above scenario is qualitatively very similar to what is obtained in Ref. [29], where a BoseFermi mixture is analyzed by means of more precise but much more numerically demanding quantum Monte Carlo simulations. The four phases, with a sufficiently large value of $U_{2}$ instead of an infinitely large one, have been proven to be stable under small variations of the interaction parameters.

A very interesting issue recently raised by Roscilde and Cirac [26] concerns the possibility that strongly-interacting mixtures exhibit a complex low-energy landscape challenging very efficient relaxation dynamics such as that inherent in quantum Monte Carlo simulations. As a result, usually efficient minimization algorithms fail to converge to the configuration attaining the minimum energy, and get virtually trapped into low-energy quantum emulsion states consisting of a random arrangement of droplets characterized by different phases of the two atomic species. The same phenomenology is captured by the Gutzwiller mean-field approach, as discussed in Refs. [27] and [30] for strongly interacting bosonic mixtures and single-species bosons with long range dipolar interactions, respectively.

For interspecies interactions exceeding the phase-separation threshold, $U_{1,2}^{\prime \prime}$ the minimization algorithm we adopt gets trapped in quantum emulsion states, which exhibit different features in regions 3 and 4 of the phase diagram in Fig. 1, In order to illustrate this, we discuss the properties of the quantum emulsion configurations at two representative values of the interspecies interaction, $U_{1,2}=1.1$ and $U_{1,2}=1.35$. We first of all notice that, as discussed in Refs. 26 27. the energy of quantum emulsion states exhibits a clear linear dependence on the number of interfaces between neighbouring droplets, as it is shown by panels b. and c. of Fig. 1. Clearly, the minimum energy is attained by a configuration featuring the lowest possible number of phase interfaces, i.e. two. We emphasize that the lowest-energy states in Fig. 11b., c. have been obtained by including an ad hoc constraint on the number of interfaces. Indeed, the presence of a large number of low-energy metastable states makes the reaching of the true ground state a very hard task.

Fig. 2 shows the appearance of the typical quantum emulsion state at $U_{1,2}=1.1$ (left) and $U_{1,2}=1.35$ (right). In both cases the upper and lower panels focus on soft- and hard-core bosons, respectively. The solid black line corresponds to the local density $\left\langle n_{f, j}\right\rangle$, whereas the coloured areas represent the complex local order parameter $\alpha_{f, j}=\left|\alpha_{f, j}\right| e^{i \varphi_{f, j}}=\left\langle a_{f, j}\right\rangle$. More precisely, the height of such areas is determined by $\left|\alpha_{f, j}\right|^{2}$ whereas the colors denote the phase 

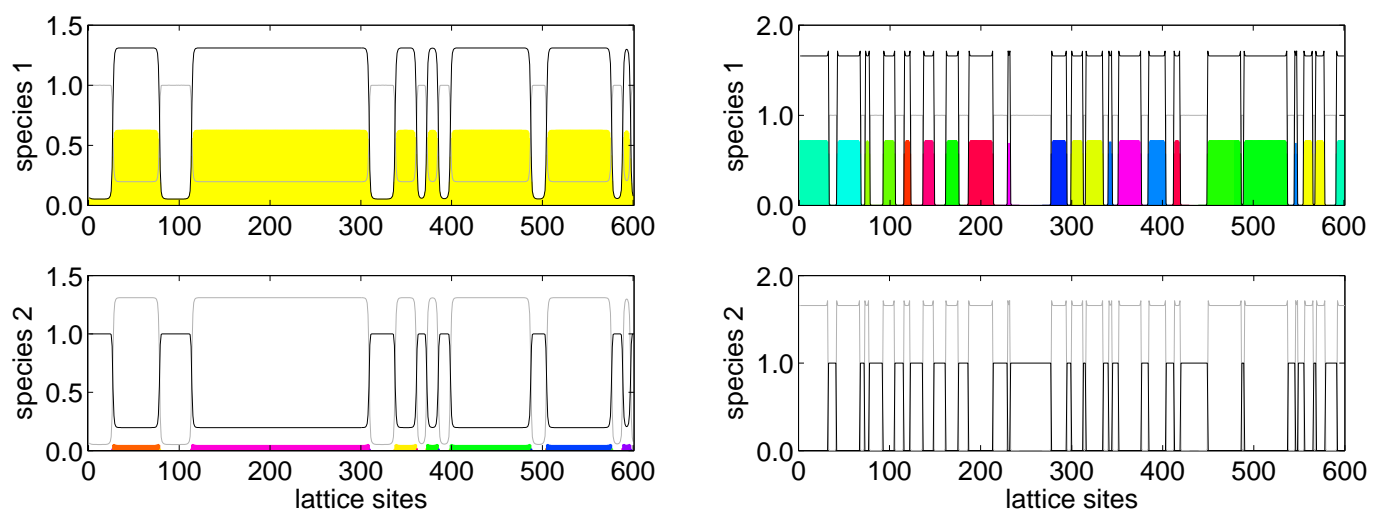

Fig. 2. Typical quantum emulsion states in regions 3 (left, $U_{1,2}=1.1$ ) and 4 (right, $U_{1,2}=1.35$ ) of the phase diagram in Fig. 1 a. In both cases the top and bottom panels focus on the soft- and hard-core bosons, respectively. The black solid line represents the local site occupation $\left\langle n_{f, j}\right\rangle$, whereas the coloured areas are related to the complex local order parameter, $\left\langle a_{f, j}\right\rangle=\left|\alpha_{f, j}\right| e^{i \varphi_{f, j}}$. Specifically, the height of the area is $\left|\alpha_{f, j}\right|^{2}$, while the color is associated to the phase $\varphi_{f, j}$ through the colorbar in Fig. 1]d.

$\varphi_{f, j}$ according to the colorbar in Fig. 1 $\mathrm{d}$. We note that each droplet can be characterized by an unique value of the phase, which can vary arbitrarily from droplet to droplet. A light gray solid line representing the local density of the species analyzed in detail in the other panel is also drawn for comparison in each panel. In both cases one can recognize the presence of "droplets" of two kinds.

For $U_{1,2}=1.1$ the hard-core species comprises irregularly interleaved superfluid and insulating domains, which prevents a superfluid flow. Indeed, on a one dimensional system such as the one under investigation, $\alpha_{2, j}$ must be finite at every site in order to produce a nonzero $F_{\mathrm{s}}^{(2)}$ [42]. Hence the hard-core bosons are in a kind of glassy insulator, as we discuss in more detail below. Conversely, the phase of soft-core bosons could be defined as a disordered superfluid. Indeed it also consists of irregularly interleaved domains, which however always exhibit a superfluid character since the value of $\alpha_{i, 2}$ never vanishes.

For $U_{1,2}=1.35$ the two atomic species never occupy the same lattice site, i.e. they are completely phase separated. Mott-like hard-core droplets are randomly intermingled with superfluid soft-core domains. Hence in this case the soft-core species is in a Bose-glass-like phase.

In both the examined cases the droplets composing the quantum emulsion are arranged in a random fashion. This feature is quite interesting, and demonstrates the spontaneously disordered nature of the low energy configurations in strongly interacting atomic mixtures [26]. Recall indeed that the original Hamiltonians do not contain any explicit disorder source. Even ignoring the configurations which are equivalent through a lattice translation, a virtually infinite number of spatial arrangements of droplets are possible with essentially the same energy, very close to the ground state. Furthermore, in cases where $\alpha_{f, j}$ is finite only inside one of the two classes of droplets - as for species 2 at $U_{1,2}=1.1$ and for species 1 at $U_{1,2}=1.35-$, a further source of energy degeneracy lies in the phase $\varphi_{f, j}$ of the order parameter. Note indeed that in the above mentioned cases such phase has a constant value within each droplet, but varies across different droplets. This is explained by observing that each droplet can be well approximated as a virtually isolated and almost uniform system, owing to the vanishing of the order parameter in the surrounding droplets. In this situation one expects the chosen periodic boundary conditions to be irrelevant. In a uniform system an overall constant phase has no effect on the observables, and in particular on the system energy. In the present case different droplets have in general a different phase, which manifests itself in observables involving different lattice sites, e.g. like

$$
\left\langle a_{f, j}^{\dagger} a_{f, \ell}\right\rangle=\left|\alpha_{f, j}\right|\left|\alpha_{f, \ell}\right| e^{i\left(\varphi_{f, \ell}-\varphi_{f, j}\right)}
$$


This has virtually no effect on the energy, since it gets contributions from terms where $\ell$ and $j$ are nearest neighbours. Indeed when both sites belong to the same droplet the equal phases cancel out, while when the sites are on different sides of a droplet boundary the possible phase difference is canceled by the virtual vanishing of the order parameter outside the droplet. It is also clear that small variations of the phases $\varphi_{f, j}$ result in an increase of the energy, which demonstrates the local-minimum character of these configurations 43 .

Interestingly, the different phases of the droplets can play an important role in the experimental measure of the system coherence. This is usually obtained by imaging the ultracold atomic cloud after a few milliseconds of free expansion. The interference pattern in the absorption image is substantially described by the Fourier transform of the one-body density matrix summarizing the two-site correlations [41], Eq. (8), whose mean-field form is

$$
S_{f}(\mathbf{k})=\frac{1}{M} \sum_{j}\left(n_{f, j}-\left|\alpha_{f, j}\right|^{2}\right)+\frac{1}{M}\left|\sum_{j} e^{i\left(\mathbf{k} \cdot \mathbf{r}_{j}-\varphi_{f, j}\right)}\right| \alpha_{f, j}||^{2}
$$

We once again recall that in the homogeneous case the mean-field approach results in twosite correlations independent of the site distance, which leads to an artifact finite condensate fraction in the thermodynamic limit of one dimensional systems. On finite systems this artifact is less serious, since the exact result is anyway very slowly decaying with inter-site distance 37. For instance, in both the mean-field and exact cases $S_{f}(\mathbf{k})$ exhibits a large peak at $\mathbf{k}=0$ surrounded by small secondary peaks. Therefore the (incipient) long-range order predicted by the mean-field approach turns out to be a reasonable approximation to the expected quasilong-range order on homogeneous systems.

In the case of single a quantum emulsion state such as those depicted in Fig. 2 the correlations in Eq. (9) would be also finite at arbitrary distance, however modulated by a phase factor. This is in contrast to what one would expect in a disordered, strongly interacting regime. Nevertheless, the expected short range correlations are to some extent captured by our mean-field approximation. Indeed, it seems reasonable to assume that the state of the system consists of a superposition of many almost-degenerate low-energy quantum emulsion states. These will be characterized by different phase-interface arrangements compatible with the typical droplet size dictated by the system parameters, as well as by different phases of the local order parameter within each droplet. This results in $\rho_{i j} \approx\left|\alpha_{i}\right|\left|\alpha_{j}\right|=\left|\alpha_{i}\right|^{2}>0$ for sites $i$ and $j$ belonging to the same droplet, and $\rho_{i j}=0$ otherwise, which shows that the correlation lenght is comparable with the average droplet size, as expected. When this size is of the order of a few lattice sites we expect the absorption image of a quantum emulsion phase to be hardly distinguishable from that of a Mott-insulating phase. This presents some analogies with the case of the Bose-glass phase induced in a single-species system by (quasi)random local potentials [5.44.

\section{Conclusion}

In summary, we have discussed the occurrence of four different phases in a 1D lattice bosonic mixture as a result of the increase of a single parameter, i.e. the interspecies repulsion. The features of the different phases have been analyzed by employing suitable observables, and special attention has been devoted to the quantum emulsion metastable states occurring at large value of the interspecies repulsion [26|27. Also, we discussed experimental issues such as the visibility of these quantum emulsion states, which could greatly reduced by the randomness of the phase of the order parameter within each isolated droplet. Owing to the large parameter space, we expect that further quantum phases can crop out in different regimes. For example, we verified that for a sufficiently large increase in the hopping amplitudes phase 3 is replaced by a different quantum emulsion where both species behave as a disordered fluid. Such complex phases and the effect of the dimensionality on phase segregation will be the subject of future work. 


\section{Acknowledgments}

The authors acknowledge fruitful discussions with Tommaso Roscilde.

\section{References}

1. F. D. M. Haldane, Phys. Lett. A 80, 280 (1980).

2. M. P. Fisher, P. B. Weichman, G. Grinstein, and D. S. Fisher, Phys. Rev. B 40, 546 (1989).

3. M. Greiner, O. Mandel, T. Esslinger, T. W. Hänsch, and I. Bloch, Nature 415, 39 (2002).

4. D. Jaksch, C. Bruder, J. I. Cirac, C. W. Gardiner, and P. Zoller, Phys. Rev. Lett. 81, 3108 (1998).

5. J. E. Lye, L. Fallani, M. Modugno, D. S. Wiersma, C. Fort, and M. Inguscio, Phys. Rev. Lett. 95, 070401 (2005).

6. D. Clément, A. F. Varón, M. Hugbart, J. A. Retter, P. Bouyer, L. Sanchez- Palencia, D. M. Gangardt, G. V. Shlyapnikov, and A. Aspect, Phys. Rev. Lett. 95, 170409 (2005).

7. U. Gavish and Y. Castin, Phys. Rev. Lett. 95, 020401 (2005).

8. S. Ospelkaus, C. Ospelkaus, O. Wille, M. Succo, P. Ernst, K. Sengstock and K. Bongs, Phys. Rev. Lett. 96, 180403 (2006).

9. B. Damski, L. Santos, E. Tiemann, M. Lewenstein, S. Kotochigova, P. Julienne, and P. Zoller, Phys. Rev. Lett. 90, 110401 (2003).

10. A. J. Daley, P. O. Fedichev, and P. Zoller, Phys. Rev. A 69, 022306 (2005).

11. A. B. Kuklov and B. V. Svistunov, Phys. Rev. Lett. 90, 100401 (2003).

12. L.-M. Duan, E. Demler, and M. D. Lukin, Phys. Rev. Lett. 91, 090402 (2003).

13. F. Ferlaino, E. De Mirandes, R. Heidemann, G. Roati, G. Modugno and M. Inguscio, J. Phys. IV 116253 (2004).

14. K. Günter, T. Stöferle, H. Moritz, M. Köhl and T. Esslinger, Phys. Rev. Lett. 96, 180402 (2006).

15. J. Catani, L. De Sarlo, G. Barontini, F. Minardi, and M. Inguscio, Phys. Rev. A 77, 011603R (2008).

16. A. Albus, F. Illuminati and J. Eisert, Phys. Rev. A 68, 023606 (2003)

17. S. K. Adhikari, Phys. Rev. A 70043617 (2004);

18. F. Illuminati and A. Albus, Phys. Rev. Lett. 93, 090406 (2004)

19. M. Cramer, J. Eisert, and F. Illuminati, Phys. Rev. Lett. 93, 190405 (2004).

20. R. Roth and K. Burnett, Phys. Rev. A 68, 023604 (2003).

21. R. Roth and K. Burnett, Phys. Rev. A 69, 021601(R) (2004)

22. E. Altman, W. Offstetter, E. Demler and M. D. Lukin, New J. Phys. 5, 113 (2003).

23. G.-H. Chen and Y.-S. Wu, Phys. Rev. A 67, 013606 (2003).

24. O. E. Alon, A. I. Streltsov and L. S. Cederbaum, Phys. Rev. Lett 97, 230403 (2006)

25. L. Mathey, Phys. Rev. B 75, 144510 (2007).

26. T. Roscilde and J. I. Cirac, Phys. Rev. Lett. 98, 190402 (2007).

27. P. Buonsante, S.M. Giampaolo, F. Illuminati, V. Penna and A. Vezzani, Phys. Rev. Lett. 100, $240402(2008)$

28. L. Pollet, M. Troyer, K. Van Houcke and S. M. A. Rombouts, Phis. Rev. Lett. 96, 190402 (2006).

29. F. Hébert, F. Haudin, L. Pollet, and G. G. Batrouni, Phys. Rev. A. 76, 043619 (2007).

30. C. Menotti, C. Trefzger, and M. Lewenstein, Phys. Rev. Lett. 98, 235301 (2007).

31. K. V. Krutitsky and R. Graham, Phys. Rev. A 70063610 (2004);

32. K. V. Krutitsky, M. Timmer and R. Graham, Phys. Rev. A 71, 033623 (2005);

33. M. Yamashita and M. W. Jack, Phys. Rev. A 76, 023606 (2007).

34. A. Argüelles and L. Santos, Phys. Rev. A 75, 053613 (2007).

35. P. Buonsante and V. Penna, J. Phys. A 41, 175301 (2008).

36. A. Isacsson, M. -C. Cha, K. Sengupta and S. M. Girvin, Phys. Rev. B 72, 184507 (2005).

37. See e.g. F.D.M Haldane, Phys. Rev. Lett. 47, 1840 (1981); C. Kollath, U. Schollwöck, J. von Delft, and W. Zwerger, Phys. Rev. A 69, 031601(R) (2004); D.M. Gangardt and G.V. Schlyapnikov, New Journal of Physics 8, 167 (2006); E. Toth, A.M. Rey, P.B. Blakie, Phys. Rev. A 78, 013627 (2008).

38. B.S. Shastry and B. Sutherland, Phys. Rev. Lett. 65, 243 (1991).

39. D. J. Scalapino, S. R. White, and S. Zhang, Phys. Rev. B 47, 7995 (1993).

40. R. E. Peierls, Z. Phys. 80, 763 (1933).

41. F. Gerbier, A. Widera, S. Fölling, O. Mandel, T. Gericke and I. Bloch, Phys. Rev. A 72053606 (2005). 
42. P. Buonsante, V. Penna, A. Vezzani, and P. B. Blakie, Phys. Rev. A 76, 011602(R) (2007)

43. Rigorously speaking, aligning all the phases of the local order parameters while leaving their moduli unchanged attains the minimum energy for a given droplet arrangement. This shows that states where different droplets are characterized by different phases cannot be the absolute minumum. On the other hand, as we discussed above, a generic quantum emulsion state cannot be the absolute minimum either, because of its many phase interfaces.

44. P. Buonsante, F. Massel, V. Penna and A. Vezzani, Laser Physics 18, 653 (2008). 